\title{
Interpolating View and Scene Motion by Dynamic View Morphing
}

\author{
Russell A. Manning \\ Department of Computer Sciences \\ University of Wisconsin \\ Madison, Wisconsin 53706 \\ \{rmanning,dyer\}@cs.wisc.edu
}

Charles R. Dyer

\begin{abstract}
We introduce the problem of view interpolation for dynamic scenes. Our solution to this problem extends the concept of view morphing [11] and retains the practical advantages of that method. We are specifically concerned with interpolating between two reference views captured at different times, so that there is a missing interval of time between when the views were taken. The synthetic interpolations produced by our algorithm portray one possible physically-valid version of what transpired in the scene during the missing time. It is assumed that each object in the original scene underwent a series of rigid translations. Dynamic view morphing can work with widely-spaced reference views, sparse point correspondences, and uncalibrated cameras. When the camera-to-camera transformation can be determined, the synthetic interpolation will portray scene objects moving along straightline, constant-velocity trajectories in world space.
\end{abstract}

\section{Introduction}

View interpolation [4] involves creating a series of virtual views of a scene that, taken together, represent a continuous and physically-correct transition between two reference views of the scene. Previous work on view interpolation has been restricted to static scenes. Dynamic scenes change over time and, consequently, these changes will be evident in two reference views that are captured at different times. Therefore, view interpolation for dynamic scenes must portray a continuous change in viewpoint and a continuous change in the scene itself in order to transition smoothly between the reference views (Fig. 1).

Our approach to this problem is based upon an earlier technique called view morphing [11], which provides a method for interpolating between two widelyspaced views of a static scene. The technique has sev-

The support of NSF under grant IIS-9530985 and DARPA under agreement F30602-97-1-0138 is gratefully acknowledged. eral strengths that make it suitable for practical applications. First, only two reference views are assumed. Second, it does not require that camera calibration be provided nor does it need to calculate the camera parameters. Third, the method works even when only a sparse set of correspondences between the reference views is known. If more information about the reference views is available, this information can be used for added control over the output and for increased realism.

In addition to view morphing, numerous existing methods could be used to create view interpolations for static scenes $[6,10,1,12,14]$. However, none of these methods is directly applicable to dynamic scenes. Avidan and Sashua [2] provide a method for recovering the geometry of dynamic scenes in which the objects move along straight-line trajectories. Once the geometry is recovered, dynamic view interpolations could be created using the standard graphics pipeline. However, their algorithm does not apply to the problem discussed in this paper because it assumes that five or more views are available and that the camera matrix for each view is known or can be recovered. There are several mosaicing techniques for dynamic scenes [8, 5], but mosaicing involves piecing together several small-field views to create a single large-field view, whereas view interpolation involves synthesizing new views from vantage points not in the reference set.

Because the original view morphing algorithm assumes a static scene, we refer to it as static view morphing to distinguish it from the dynamic view morphing technique presented in this paper.

We seek to perform view interpolation directly from the reference views, without additional information about the scene. Consequently, there will be a missing interval of time between when the reference views were captured, and it will be impossible to know for certain what occurred during the missing interval. It is not our goal in this work to try and deduce the most 

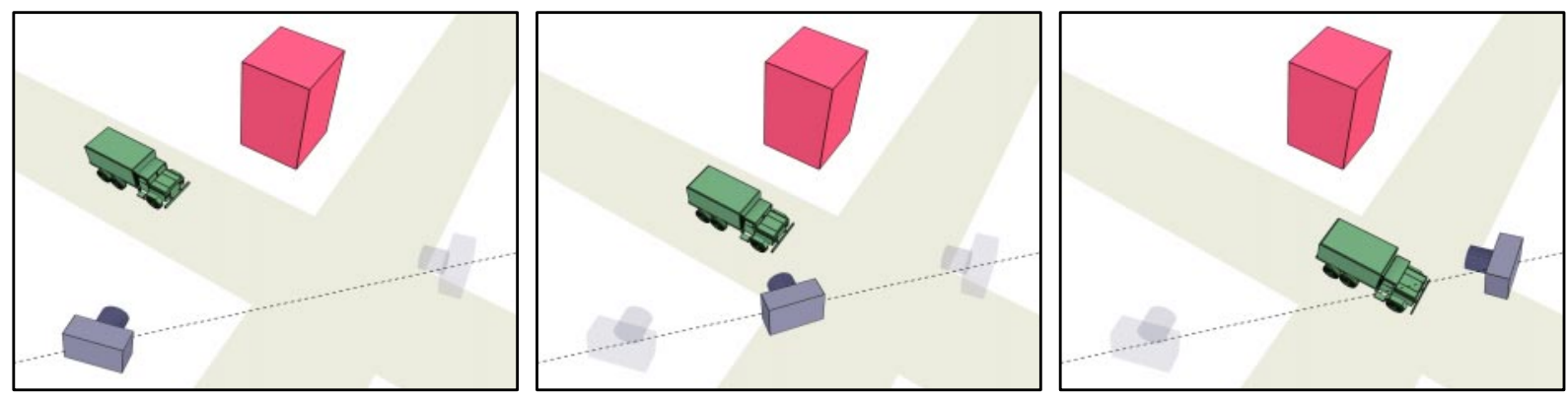

Figure 1: A dynamic scene at three different times. The goal of view interpolation for dynamic scenes is to synthesize the view from the camera in the middle frame starting with only the two reference views from the cameras in the left and right frames.

likely manner in which the scene changed. Instead, we are interested in portraying some possible way in which the scene could have changed, and we want the portrayal to be physically correct and continuous.

Our method is for dynamic scenes that satisfy the following assumption: For each object in the scene, all of the changes that the object undergoes during the missing time interval, when taken together, are equivalent to a single, rigid translation.

The term object has a specific meaning in this paper, defined by the condition given above: An object is a group of particles in a scene for which there exists a fixed vector $\mathbf{u} \in \Re^{3}$ such that each particle's total motion during the missing time interval is equal to $\mathbf{u}$.

A method for dynamic view interpolation, even if it is physically accurate, may be unsatisfactory if it portrays objects moving along unreasonable trajectories. For instance, when portraying a car driving across a bridge, it is essential that the car stay on the bridge during the entire sequence. To address this problem, we have developed techniques for portraying both straight-line motion (in a camera-based coordinate frame) and straight-line, constant-velocity motion (in camera and world coordinate frames). For brevity, we refer to the latter style of portrayal as linear motion. Fig. 1 depicts a linear motion view interpolation.

If the reference cameras share the same position in world coordinates, then the virtual camera shares that position as well and straight-line motion relative to the virtual camera also implies straight-line motion in world coordinates. However, this may not be the case if the virtual camera moves during the view interpolation, as Fig. 2 demonstrates. It is easy to show that if all objects can be portrayed undergoing linear motion in camera coordinates, then the virtual camera can be considered undergoing linear motion in world

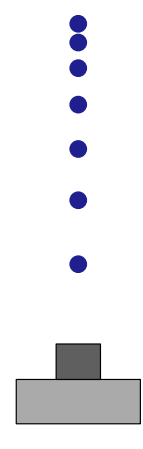

(i)

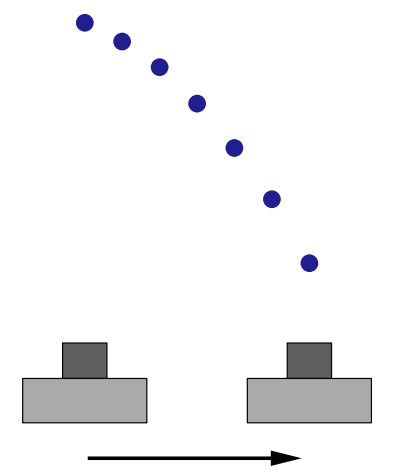

(ii)
Figure 2: (i) A round object is filmed moving along a trajectory that is a straight line in the camera's frame of reference. The object is shown at equal time intervals and does not move at constant velocity. (ii) If the camera was in motion during the filming, then the object did not follow a straight-line trajectory in world coordinates.

coordinates, in which case all the objects will undergo linear motion in world coordinates as well.

\section{Static view morphing}

Static view morphing works by first prewarping the reference views to make their image planes parallel. After the prewarp, conjugate points in the two views are linearly interpolated to produce a physicallyaccurate new view of the scene. The new locations of the conjugate points are used to guide a morphing algorithm in filling the remainder of the virtual view. Only the interpolated conjugate points are guaranteed to be viewed in the correct, physically-accurate locations. By increasing the density of conjugate points, the virtual view can be made arbitrarily accurate. The prewarp is performed from information available in the 
fundamental matrix, which is calculated directly from the conjugate points. Complete details of the algorithm can be found in [11].

\section{Dynamic view morphing}

\subsection{Preliminary concepts}

We assume the two reference views are captured at time $t=0$ and time $t=1$ through pinhole cameras, which are denoted camera $A$ and camera $B$, respectively.

We always use a fixed-camera formulation, meaning we assume that the two reference cameras are at the same location and that the world moves around them; this is accomplished by subtracting the actual displacement between the two cameras from the motion vectors of all objects in the scene. Under this assumption, the camera matrices are just $3 \times 3$ and each camera is equivalent to a basis for $\Re^{3}$. Note that no assumption is made about the cameras other than that they share the same optical center; the camera matrices can be completely different.

We let $U$ denote the "universal" or "world" coordinate frame, and use the notation $\mathcal{T}_{U A}$ to mean the transformation between basis $U$ and basis $A$. Hence $\mathcal{T}_{U A}$ is the camera matrix for $A$. Of particular interest to our work is the matrix $\mathcal{T}_{A B}$. Note that capital script letters will always represent $3 \times 3$ matrices; in particular, $\mathcal{I}$ is the identity matrix.

A position or a direction in space exists independently of what basis is used to measure it; we will use a subscript letter when needed to denote a particular basis. For instance, if $\mathbf{e}$ is the direction between two cameras (that are not at the same location), then $\mathbf{e}_{A}$ is $\mathbf{e}$ measured in basis $A$. The quantity $\mathbf{e}$ is called the epipole. The fundamental matrix $\mathcal{F}$ for two cameras $A$ and $B$ that are at different locations has the following representation [7]:

$$
\mathcal{F}=\left[\mathbf{e}_{B}\right]_{\times} \mathcal{T}_{A B}
$$

Here $[\cdot]_{\times}$denotes the cross product matrix. When the two cameras share the same optical center, the fundamental matrix is 0 and has no meaning. However, for each moving object $\Omega$ in the scene, we can define a new kind of fundamental matrix. If, after making the fixed-camera assumption, $\Omega$ is moving in direction $\mathbf{u}$, then the fundamental matrix for the object is:

$$
\mathcal{F}_{\Omega}=\left[\mathbf{u}_{B}\right]_{\times} \mathcal{T}_{A B}
$$

The epipoles of $\mathcal{F}_{\Omega}$ are the vanishing points of $\Omega$ as viewed from the two reference cameras, and the epipolar lines trace out trajectories for points on $\Omega$.

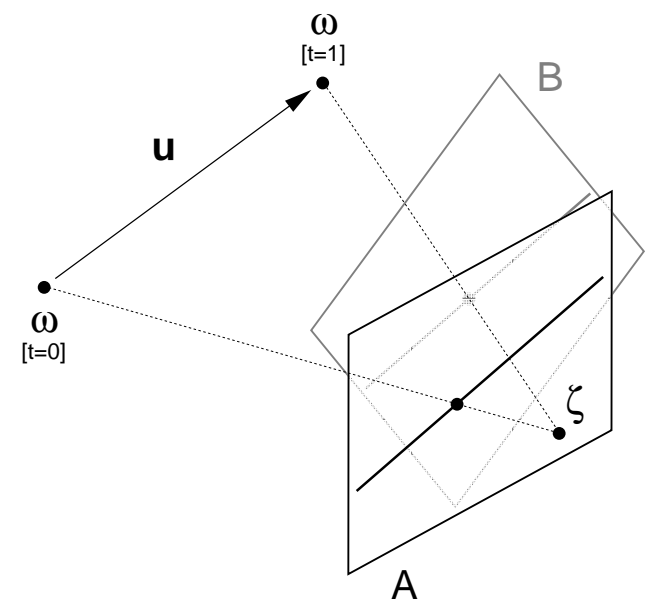

Figure 3: Cameras $A$ and $B$ share the same optical center $\zeta$ and are viewing a point on an object that translates by $u$. The image planes of the cameras are parallel to each other and to $u$, and hence interpolation will produce a physically-correct view of the object. On each image plane a line parallel to $u$ is shown.

\subsection{View interpolation for a single mov- ing object}

Assume the two reference cameras share the same optical center and are viewing a point $\omega$ that is part of an object $\Omega$ whose translation vector is $\mathbf{u}$. Let $\mathbf{q}$ and $\mathbf{q}+\mathbf{u}$ denote the position of $\omega$ at time $t=0$ and $t=1$, respectively (Fig. 3).

Assume for this subsection that the image planes of the cameras are parallel to each other and to $\mathbf{u}$. The first half of this condition means that the third row of $\mathcal{T}_{U A}$ equals the third row of $\mathcal{T}_{U B}$ scaled by some constant $\lambda$. The second half means that $\left(\mathcal{T}_{U A} \mathbf{u}_{U}\right)_{z}=$ $\left(\mathcal{T}_{U B} \mathbf{u}_{U}\right)_{z}=0$, where $(\cdot)_{z}$ denotes the $z$-coordinate of a vector. Note that the condition can be met retroactively by using standard rectification methods [11, 9]; this is part of "prewarping" the reference views as mentioned in Section 2.

Setting $\xi=\left(\mathcal{T}_{U A} \mathbf{q}_{U}\right)_{z}=\lambda\left(\mathcal{T}_{U B}(\mathbf{q}+\mathbf{u})_{U}\right)_{z}$, the linear interpolation of the projection of $\omega$ into both cameras is

$$
(1-s) \frac{1}{\xi} \mathcal{T}_{U A} \mathbf{q}_{U}+s \frac{\lambda}{\xi} \mathcal{T}_{U B}(\mathbf{q}+\mathbf{u})_{U}
$$

Now define a virtual camera $V$ by the matrix

$$
\mathcal{T}_{U V}=(1-s) \mathcal{T}_{U A}+s \lambda \mathcal{T}_{U B}
$$

Then the linear interpolation (3) is equal to the projection of scene point $\mathbf{q}(s)$ onto the image plane of 


\begin{tabular}{|c|c|}
\hline if prewarps make... & then interpolation is... \\
\hline image planes parallel & physically correct \\
\hline $\begin{array}{c}\text {...and conjugate directions } \\
\text { equal up to a scalar }\end{array}$ & $\begin{array}{c}\text {..and depicts straight-line } \\
\text { motion }\end{array}$ \\
\hline ...and the scalar is $\lambda$ & $\begin{array}{c}\text {...and the motion is } \\
\text { constant-velocity }\end{array}$ \\
\hline
\end{tabular}

Figure 4: How the interpolation sequence is related to different preconditions on the reference views. Stricter preconditions lead to increased control over the output.

camera $V$, where

$$
\begin{aligned}
\mathbf{q}(s) & =\mathbf{q}+\mathbf{u}(s) \\
\mathbf{u}(s)_{V} & =s \lambda \mathbf{u}_{B}
\end{aligned}
$$

Notice that $\mathbf{u}(s)$ depends only on $\mathbf{u}$ and the camera matrices and not on the starting location q. Thus linear interpolation of conjugate object points by a factor $s$ creates a physically-valid view of the object. The object is seen as it would appear through camera $V$ if it had been translated by $\mathbf{u}(s)$ from its starting position.

Note that in Eq. 6, $\mathbf{u}(s)$ is represented in basis $V$. Since $V$ changes with $s$ it is difficult in general to characterize the trajectory in world coordinates. To have greater control over the interpolation process, we now prove that straight-line motion is achieved when $\mathbf{u}_{A}=\mathbf{u}_{B}$ up to an arbitrary scalar, and constantvelocity straight-line motion (i.e., linear motion) is achieved when $\mathbf{u}_{A}=\lambda \mathbf{u}_{B}$ (Fig. 4):

Assume $\mathbf{u}_{A}=k \mathbf{u}_{B}$ for some scalar $k$. Multiplying both sides of Eq. 4 on the right by $\mathcal{T}_{B U}$ yields

$$
\mathcal{T}_{B V}=(1-s) \mathcal{T}_{B A}+s \lambda \mathcal{I}
$$

By multiplying both sides of Eq. 7 on the right by $\mathbf{u}_{B}$ and on the left by $\mathcal{T}_{V B}$ the following can be derived:

$$
\mathcal{T}_{V B} \mathbf{u}_{B}=\frac{1}{(1-s) k+s \lambda} \mathbf{u}_{B}
$$

Multiplying both sides of Eq. 6 by $\mathcal{T}_{V B}$ now yields:

$$
\mathbf{u}(s)_{B}=\frac{s \lambda}{(1-s) k+s \lambda} \mathbf{u}_{B}
$$

The basis $V$ no longer plays a role and the virtual trajectory, given by $\mathbf{u}(s)_{B}$, is a straight-line in basis $B$. If $k=\lambda$ then $\mathbf{u}(s)_{B}=s \mathbf{u}_{B}$ and the virtual object moves at constant velocity. The results are in basis $B$, but multiplying by $\mathcal{T}_{B U}$ or $\mathcal{T}_{B A}$ indicates that the results

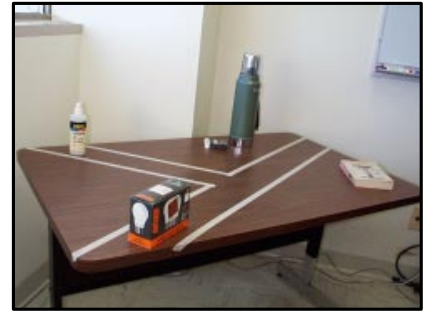

original view

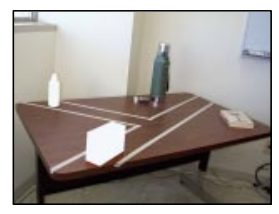

layer 0 (background)

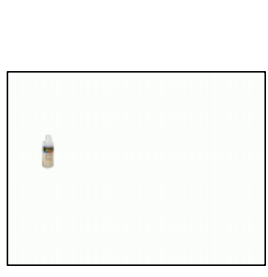

layer 1

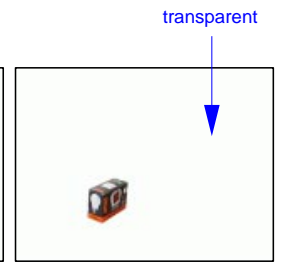

layer 2
Figure 5: A view divided into layers. Each layer corresponds to a moving object. The single "background" object contains many different objects that all translate by the same amount.

also hold in world coordinates and camera $A$ 's coordinates, thus completing the proof. Keep in mind that the world coordinate system used in this context has its origin at the shared optical center of the reference cameras.

If $\mathcal{T}_{B A}$ is known then the camera matrix for $B$ can be transformed into the camera matrix for $A$. This allows the view from camera $B$ at time $t=1$ to be transformed into the view from camera $A$ at time $t=1$, thus producing two views of the scene from camera $A$ at different times. For this reason, we call $\mathcal{T}_{B A}$ the camera-to-camera transformation. By applying the earlier results to this special case, we derive the following corollary which forms the basis of the algorithm in Section 3.3:

If both camera matrices are equal and if $\left(\mathcal{T}_{U A} \mathbf{u}\right)_{z}=0$, then the camera matrix for the virtual camera $V$ is just $\mathcal{T}_{U A}$ and, because $\lambda=1$ and $\mathbf{u}_{A}=\mathbf{u}_{B}$, the virtual object moves at constant velocity along a straight-line path.

\subsection{Linear motion dynamic view morph- ing algorithm}

We now present a dynamic view interpolation algorithm that will portray linear motion. The algorithm requires knowledge of $\mathcal{T}_{A B}$.

(Step 1) Segment both views into layers, with each layer representing a different moving object. Order the layers from nearest object to farthest object (Fig. $5)$. 
(Step 2) Transform each layer of view $B$ by $\mathcal{T}_{B A}$, thus creating a view from camera $A$.

(Step 3) Apply static view morphing to each layer separately.

(Step 4) Recombine the new, virtual layers in the correct depth order.

(Step 5) (Optional) Postwarp the new view.

In step (3), the virtual camera will be the same for each layer by the corollary of the previous section. Furthermore, each layer will portray its corresponding object undergoing linear motion. Consequently, step (4) produces the desired linear portrayal of the entire scene.

\subsection{Special case: parallel motion}

In this and the following section we examine some special-case scenarios for which dynamic view interpolations can be produced without knowledge of $\mathcal{T}_{A B}$.

Assume a fixed-camera formulation and let $\mathbf{u}_{i}$ denote the displacement between the position of object $i$ at time $t=0$ and its position at time $t=1$. We will say the scene consists of parallel motion if all the $\mathbf{u}_{i}$ are parallel in space.

Dynamic view morphing algorithm for parallel motion case: Segment each view into layers corresponding to objects. Apply static view morphing to each layer and recomposite the results.

The algorithm works because the fundamental matrix with respect to each object is the same, so the same prewarp works for each layer. The prewarp will make the direction of motion for each object be parallel to the $x$-axis in both views; consequently, the virtual objects will follow straight-line trajectories as measured in the camera frame. If we assume that the background object has no motion in world coordinates, then the virtual camera moves parallel to the motion of all the objects and hence the virtual object motion is straight-line in world coordinates.

\subsection{Special case: planar parallel motion}

We now consider the case in which all the $\mathbf{u}_{i}$ are parallel to some fixed plane in space. Note that this does not mean all the objects are translating in the same plane. Also note that this case applies whenever there are two moving objects.

Recall that in Section 3.2 the only requirement for the virtual view to be a physically-accurate portrayal of an object that translates by $\mathbf{u}$ is that the image planes of both reference views be parallel to $\mathbf{u}$ and to each other. In the planar parallel motion case, it is possible to prewarp the reference views so that their image planes are parallel to each other and to the displacements of all the objects simultaneously.
Dynamic view morphing algorithm for planar parallel motion case: Segment each view into layers corresponding to objects. For each reference view, find a single prewarp that sends the $z$ coordinate of the vanishing point of each object to 0 . Using this prewarp, apply static view morphing to each layer and recomposite the results.

The algorithm given above only guarantees physical correctness, not straight-line or linear motion. The appearance of straight-line motion can be created by first making the conjugate motion vectors parallel during the prewarp step [9].

\subsection{Dynamic scene hierarchy}

This section interrelates the algorithms of the previous three sections. As always, we assume a fixedcamera formulation, meaning we choose to interpret the two reference views as having been captured by cameras that shared the same optical center.

Consider classifying each object in the scene based on the direction of its translation vector, with two objects being placed in the same class if their translation vectors are parallel. A natural hierarchy emerges based on the number of distinct parallel motion classes the scene contains.

First consider scenes that have only one motion class. If the class corresponds to the null direction vector, then the scene is static and view interpolation reduces to mosaicing. If the direction vector is non-null, view interpolations can be produced via the parallel motion algorithm (Section 3.4).

When the scene has two motion classes, the planarparallel motion algorithm applies (Section 3.5). With four or more motion classes, $\mathcal{T}_{A B}$ can be determined as described in Section 4 from the four directions associated with the classes, and the linear motion algorithm applies (Section 3.3). For scenes with exactly three motion classes, either the planar-parallel algorithm applies or else $\mathcal{T}_{A B}$ can be approximated after making reasonable assumptions about the reference cameras [9].

\subsection{Affine cameras}

The mathematical development for affine cameras, which includes orthographic cameras, is similar to that for pinhole cameras. However, except in special cases, no camera-to-camera transformation exists between the reference cameras. Hence it is typically impossible to guarantee linear motion for the virtual objects. On the other hand, interpolation of conjugate points always produces a physically-valid virtual view, without needing to make the image planes parallel. Prewarps can be applied to align conjugate directions and thus achieve straight-line motion. However, in general it is 
only possible to align at most three conjugate directions. For a complete discussion, see [9].

\section{Finding $\mathcal{T}_{A B}$}

The problem of determining $\mathcal{T}_{A B}$ is central to the linear motion algorithm of Section 3.3. $\mathcal{T}_{A B}$ can be determined from four conjugate directions by a wellknown result used in mosaicing [13] (because conjugate directions become conjugate points if we treat the reference cameras as being co-centered).

If the fundamental matrix can be determined for two objects in the scene and if the objects are not moving parallel to each other, then $\mathcal{T}_{A B}$ can be determined directly from these two fundamental matrices. The previous fact is proven in [9], which also gives a method for approximating $\mathcal{T}_{A B}$ from two conjugate directions by making a reasonable assumption about the internal parameters of typical cameras.

\section{Applications}

Dynamic view morphing has many potential applications; we list a few here: filling a missing gap in a movie, creating a "hand-off" sequence to switch from one camera view to another, creating virtual views of a scene, removing obstructions or moving objects from a sequence, adding synthetic moving objects to real scenes, projecting motion into the future or past, stabilizing and compressing movie sequences, and creating movies from still images.

\section{Experimental results}

We have tested the concepts of this paper on a variety of scenarios. Fig. 6 shows the results of three tests, each as a series of still frames from a view interpolation sequence. The left-most and right-most frames of each strip are the original reference views, while the center two frames are virtual views created by the algorithm.

To create each sequence, two preprocessing steps were performed manually. First, the two reference views were divided into layers corresponding to the moving objects. Second, for each corresponding layer a set of conjugate points between the two views was determined. Since our implementation uses the BeierNeely algorithm [3] for the morphing step, we actually determined a series of line-segment correspondences instead of point correspondences. For each sequence, between 30 and 50 line-segment correspondences were used (counting every layer).

For all the sequences, the camera calibration was completely unknown, the focal lengths were different, and the cameras were at different locations.

The first sequence is from a test involving three moving objects (counting the background object).
Since $\mathcal{T}_{A B}$ could only be approximated, the appearance of straight-line motion was achieved by aligning the conjugate directions of motion for each object during the prewarp step [9]. An object's direction of motion is given by the epipoles of the object's fundamental matrix. Instead of calculating the objects' fundamental matrices, we determined the epipoles directly from the vanishing points of the tape "roads."

The second sequence involves two moving objects (counting the background object) and a dramatic change in focal length. The third sequence demonstrates the parallel motion algorithm (Section 3.4). The scene is actually static, but the pillar in the foreground and the remaining background elements are treated as two separate objects that are moving parallel to each other.

\section{Conclusion}

We have presented a method for interpolating between two views of a dynamic scene. The method requires that, for each object in the scene, the movement that occurs between the first and second views must be equivalent to a rigid translation. The algorithm produces virtual views that portray one version of what might have occurred in the scene. It is only necessary that the image planes of the reference cameras be parallel to each other and to the motion of an object for the interpolated view of the object to be physically correct. With more conditions on the reference cameras, the object can be portrayed moving along a straight-line path and even moving at constant velocity along a straight-line path. Interpolated views of a complete dynamic scene can be created by separately creating interpolated views of the scene's component objects and then combining the results.

By choosing to interpret the views as coming from the same position in space, a single theory has been created which applies to many different possible situations. In particular, the same theory applies whether or not the original reference cameras were actually cocentered. Since it is impossible to know from the reference views themselves how the original reference cameras were positioned relative to each other, the fixedcamera formulation is a natural default assumption. The virtual camera can be chosen to move along any trajectory; the choice simply alters the interpretation of the virtual views. The fixed-camera formulation also allows for a simple and intuitive development of the underlying mathematics of the theory.

Finally, it has been shown that each object in a dynamic scene has a corresponding fundamental matrix as long as the assumption of translational motion holds. From two such (distinct) fundamental matri- 

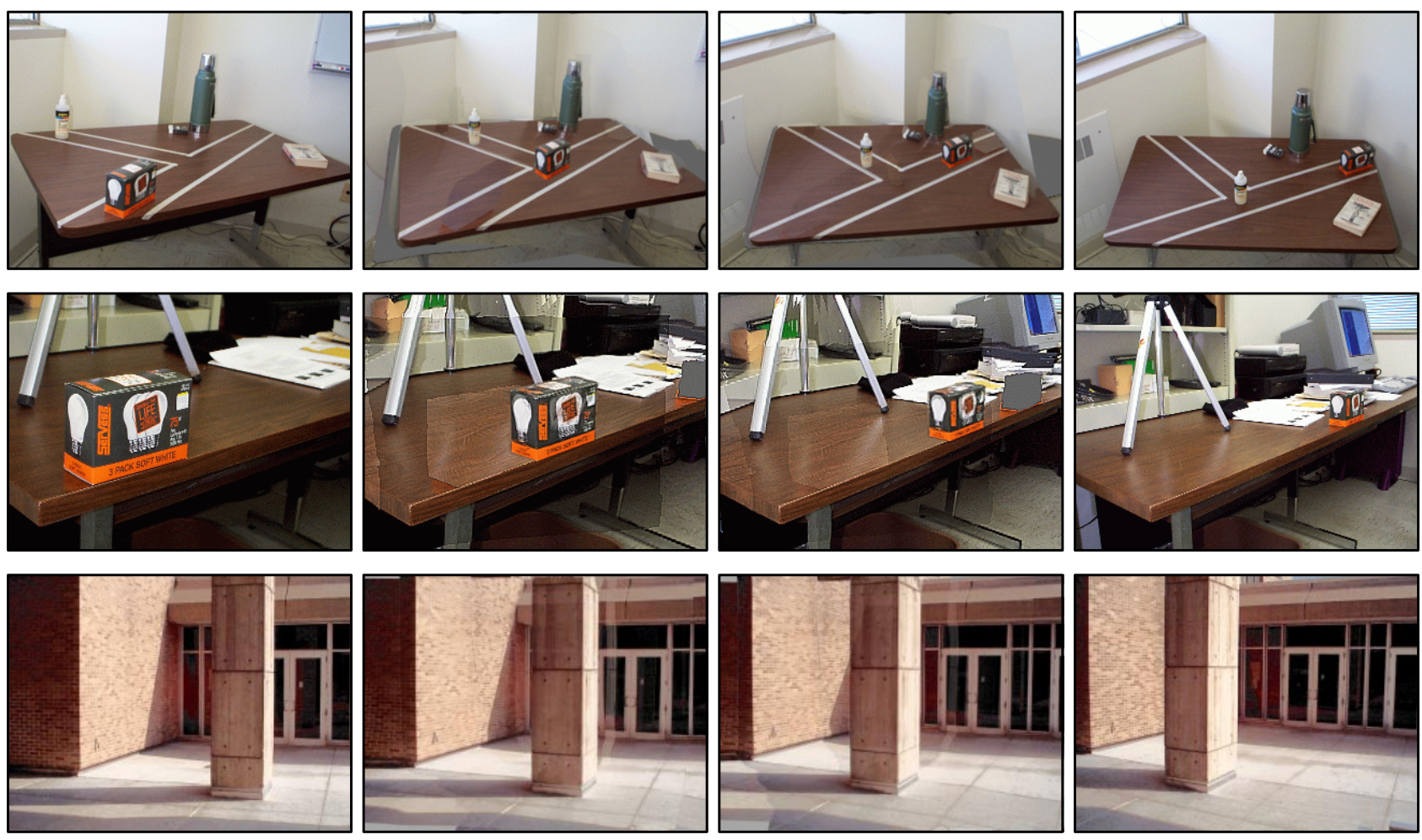

Figure 6: Experimental results.

ces, the camera-to-camera transformation can be determined.

The topics of this paper, as well as many additional topics and observations, are discussed in much greater detail in [9].

\section{References}

[1] Shai Avidan and Amnon Shashua. Novel view synthesis in tensor space. In Proc. Computer Vision and Pattern Recognition Conf., pages 1034-1040, 1997.

[2] Shai Avidan and Amnon Shashua. Non-rigid parallex for 3D linear motion. In Proc. Image Understanding Workshop, pages 199-201, 1998.

[3] Thaddeus Beier and Shawn Neely. Feature-based image metamorphosis. In Proc. SIGGRAPH 92, pages 35-42, 1992.

[4] Shenchang Eric Chen and Lance Williams. View interpolation for image synthesis. In Proc. SIGGRAPH 93, pages 279-288, 1993.

[5] James Davis. Mosaics of scenes with moving objects. In Proc. Computer Vision and Pattern Recognition Conf., pages 354-360, 1998.

[6] Olivier D. Faugeras. What can be seen in three dimensions with an uncalibrated stereo rig. In Proc. Second European Conf. on Computer Vision, pages 563-578, 1992.
[7] Richard I. Hartley. Projective reconstruction and invariants from multiple images. IEEE Trans. Pattern Analysis and Machine Intell., 16(10):1036-1041, 1994.

[8] Michal Irani, P. Anandan, and Steve Hsu. Mosaic based representations of video sequences and their applications. In Proc. Fifth Int. Conf. on Computer Vision, pages 605-611, 1995.

[9] Russell A. Manning and Charles R. Dyer. Dynamic view morphing. Technical Report 1387, Computer Sciences Department, University of WisconsinMadison, 1998.

[10] Leonard McMillan and Gary Bishop. Plenoptic modeling. In Proc. SIGGRAPH 95, pages 39-46, 1995.

[11] Steven M. Seitz and Charles R. Dyer. View morphing. In Proc. SIGGRAPH 96, pages 21-30, 1996.

[12] Steven M. Seitz and Charles R. Dyer. Photorealistic scene reconstruction by voxel coloring. In Proc. Image Understanding Workshop, pages 1067-1073, 1997.

[13] Richard Szeliski. Video mosaics for virtual environments. IEEE Computer Graphics and Applications, 16(2):22-30, 1996.

[14] Carlo Tomasi and Takeo Kanade. Shape and motion from image streams under orthography: A factorization method. Int. J. of Computer Vision, 9(2):137154, 1992. 